\title{
Proposta de apresentação da classificação dos transtornos de linguagem oral no adulto e no idoso
}

\author{
Proposal for the classification of oral language \\ disorders in the adult and elderly
}

\author{
Propuesta de presentación de la clasificación \\ de los trastornos de lenguaje oral \\ en el adulto y en el anciano
}

Bárbara Costa Beber*

\section{Resumo}

Introdução: Indivíduos adultos e idosos acometidos por lesões ou disfunções do Sistema Nervoso Central podem apresentar como consequência quadros diversos de transtornos de linguagem. Definir o tipo de transtorno de linguagem que ocorre em cada paciente tem grande relevância clínica e científica. A literatura descreve variados tipos de transtornos de linguagem oral, porém não apresenta um sistema de classificação com o maior número possível de tipos de transtornos de linguagem. Objetivo: Propor uma forma de apresentação abrangente da classificação dos transtornos de linguagem oral que podem ocorrer em adultos e idosos. Método: Revisão não sistemática de literatura. A apresentação dos transtornos de linguagem foi realizada a partir do agrupamento dos diversos tipos de transtornos de linguagem já descritos na literatura, mas com taxonomia organizada considerando critérios como a manifestação linguística e etiologia. Resultados: Os tipos de transtornos de linguagem oral que acometem a população em questão foram classificados em três grandes categorias, os transtornos de linguagem afásicos, os transtornos de linguagem não afásicos, e os transtornos cognitivos da comunicação. $\mathrm{O}$ grupo de transtornos de linguagem afásicos recebeu uma subclassificação própria. Conclusão: A utilização desta classificação poderá auxiliar a comunicação entre os profissionais, permitir a caracterização dos pacientes e facilitar o raciocínio clínico. É esperado que o avanço científico na área acarrete em mudanças na classificação destas condições e no próprio paradigma de estabelecimento dos diagnósticos médico e fonoaudiológico.

Palavras-chave: Classificação; Transtornos da linguagem; Afasia; Comunicação; Lesões encefálicas

* Universidade Federal de Ciências da Saúde de Porto Alegre - UFCSPA, Porto Alegre, RS, Brasil.

Contribuição do Autor:

$\mathrm{BCB}$ - elaboração do texto completo.

E-mail para correspondência: Bárbara Costa Beber barbaracbeber@gmail.com Recebido: 30/01/2018

Aprovado: $12 / 11 / 2018$ 
Abstract

Introduction: Adult and elderly individuals that were affected by lesion or dysfunction of the Central Nervous System may present language disorders as consequence. The definition of the language disorder type is of relevance to the clinical and scientific practices. The literature reports a variety of types of language disorders but does not present a classification system with as many types of language disorders as possible. Objective: To propose a comprehensive presentation of the classification of oral language disorders that can affect adults and elderly. Methods: Non-systematic literature review. The presentation of language disorders was made by grouping the different types of language disorders already described in the literature, but with an organized taxonomy that considered criteria such as linguistic manifestation and etiology Results: The types of oral language disorders that can occur in this population were classified into three categories: the aphasic language disorders, non-aphasic language disorders and cognitive-communication disorders. The category aphasic language disorder received its own subclassification. Conclusion: This classification might help the communication among professionals, might allow the characterization of patients, and might facilitate the clinical reasoning. It is expected that the scientific advance in this field leads to changes in the classification systems, and also in the paradigm of establishing medical and speech-language diagnoses.

Keywords: Classification; Language disorders; Aphasia; Communication; Brain injuries

\section{Resumen}

Introducción: Individuos adultos y ancianos acometidos por lesiones o disfunciones del Sistema Nervioso Central pueden presentar como consecuencia cuadros diversos de trastornos del lenguaje. Definir el tipo de trastorno de lenguaje que ocurre en cada paciente tiene gran relevancia clínica y científica. La literatura describe variados tipos de trastornos de lenguaje oral, pero no presenta un sistema de clasificación con el mayor número posible de tipos de trastornos de lenguaje. Objetivo: Proponer una forma de presentación completa de la clasificación de los trastornos de lenguaje oral que pueden ocurrir en adultos y ancianos. Método: Revisión no sistemática de literatura. La presentación de los trastornos del lenguaje fue realizada a través de la agrupación de los diversos tipos de trastornos de lenguaje ya descritos en la literatura, pero con taxonomía organizada considerando criterios como la manifestación lingüística y etiologia. Resultados: Los tipos de trastornos de lenguaje oral que afectan a la población en cuestión, se clasificaron en tres grandes categorías, los trastornos del lenguaje afásicos, los trastornos del lenguaje no afásicos, y los trastornos cognitivos de la comunicación. El grupo de trastornos de lenguaje afásicos recebió una subclasificación propia. Conclusión: La utilización de esta clasificación podrá auxiliar la comunicación entre los profesionales, permitir la caracterización de los pacientes y facilitar el raciocinio clínico. Se espera que el avance científico en el área acarrete en cambios en la clasificación de estas condiciones y en el propio paradigma de establecimiento de los diagnósticos médico y fonoaudiológico.

Palabras claves: Clasificación; Trastornos del linguaje; Afasia; Comunicación; Lesiones encefálicas

\section{Introdução}

Os transtornos da linguagem oral que acometem adultos e idosos são desordens da comunicação decorrentes de disruptura em domínios linguísticos ou cognitivos que intermediam o processamento da linguagem, em decorrência de lesão ou disfunção do Sistema Nervoso Central (SNC). Apesar disso, nem sempre é possível associar todos os quadros sintomáticos de linguagem a quadros neurológi- cos atestáveis. O conhecimento dos transtornos de linguagem oral, sua classificação, sintomas e conceitos envolvidos são fundamentais para que o fonoaudiólogo seja capaz de avaliar, diagnosticar e reabilitar a população acometida por estas condições de saúde.

Os conceitos e classificações dos transtornos de linguagem podem ser diferentes de acordo com a linha de pensamento de cada autor e podem mudar ao longo dos anos de acordo com a evolução das 
evidências científicas. A classificação das afasias clássicas, por exemplo, é considerada um "mal necessário" ", pois para o planejamento terapêutico o que mais importa é conhecer a natureza do prejuízo linguístico e não o "tipo de afasia". No entanto, a classificação dessas condições em tipos e subtipos é um modo didático de realizar o raciocínio clínico dos transtornos de linguagem e também permite a comunicação entre diferentes profissionais. A respeito da relevância para o contexto de pesquisa, a classificação sintomatológica de tais transtornos permite realizar associações entre a apresentação clínica e os achados patológicos. Essas associações são extremamente necessárias para o avanço no entendimento da fisiopatologia de doenças que ainda são pouco compreendidas, como é o caso daquelas que possuem causas neurodegenerativas. Como consequência, tais pacientes poderão ser adequadamente incluídos em ensaios clínicos para a investigação dos benefícios de terapias farmacológicas e não farmacológicas.

As primeiras descrições dos transtornos de linguagem provêm dos relatos dos médicos e anatomistas Paul Broca e Carl Wernicke. ${ }^{2}$ Suas descobertas abriram caminho para o conhecimento da neurobiologia do cérebro e dos distúrbios de linguagem. Atualmente, a neuropsicologia, que é uma ciência interdisciplinar que estuda as relações entre cérebro e cognição, tem contribuído muito para o avanço do conhecimento acerca da linguagem humana. A neuropsicologia é também uma das especialidades da Fonoaudiologia e é imprescindível para o estudo e o manejo clínico dos transtornos de linguagem no adulto e no idoso, já que estes são a manifestação comportamental de lesões ou distúrbios do SNC. ${ }^{3} \mathrm{O}$ crescente avanço nas tecnologias de neuroimagem também tem permitido um melhor entendimento dos substratos neurais dos transtornos de linguagem e, consequentemente, da neurobiologia da linguagem.

Diferentes tipos de transtornos de linguagem oral são comumente descritos em livros didáticos ou artigos científicos. Porém, é necessário um modelo de classificação que apresente o maior número possível de tipos de transtornos de linguagem. Devido a esta necessidade, este artigo de revisão não sistemática de literatura tem como objetivo propor uma forma de apresentação abrangente da classificação de todos os possíveis transtornos de linguagem oral que podem ocorrer em adultos e idosos, a fim de fundamentar a clínica fonoaudio- lógica e o ensino dos transtornos de linguagem na graduação em Fonoaudiologia.

\section{Método}

A apresentação dos transtornos de linguagem oral no adulto e no idoso foi realizada a partir do agrupamento dos diversos tipos de transtornos de linguagem já descritos anteriormente na literatura, a partir do posicionamento teórico da autora. A taxonomia das três grandes categorias de transtornos de linguagem foi organizada de acordo com a natureza do déficit de comunicação oral, se puramente linguístico ou se cognitivo, podendo, nos caso dos comprometimentos linguísticos, ser afásico ou não afásico, a depender do impacto do prejuízo linguístico. As demais subcategorias foram taxonomizadas de acordo com as manifestações linguísticas e suas etiologias.

Para descrever cada um dos tipos de transtornos de linguagem foi realizada uma revisão de literatura não sistemática, buscando artigos científicos, livros ou documentos que apresentassem conceitos e características clínicas de cada um dos tipos de transtornos. As buscas foram realizadas em bases de dados de artigos científicos (Pubmed, Scielo, Lilacs, Google Acadêmico), em livros didáticos da área e em busca livre na internet. A utilização dos termos para busca não foi padronizada, porém pode-se citar entre os termos utilizados "classificação", "transtorno de linguagem", "afasia", e "transtorno de comunicação" ("classification", "language disorder", aphasia", communication disorder"). Não se delimitou data nem idioma da publicação, e os critérios para inclusão foram flexíveis, considerando a utilidade e relevância de cada publicação para descrever cada transtorno de linguagem.

\section{Resultados e discussão}

\section{Apresentação da classificação dos transtornos de linguagem oral no adulto e no idoso}

A forma de apresentação da classificação que é proposta neste artigo engloba tipos de transtornos de linguagem oral já descritos anteriormente na literatura, porém seu diferencial é a aglutinação desses tipos em um único modelo de classificação. Esses transtornos podem ser classificados, de modo geral 
em: 1) afásicos - sendo estes subdivididos em afasias clássicas, afasias cruzadas, afasias subcorticais e afasias progressivas primárias (APPs); 2) transtornos de linguagem não afásicos; e 3) transtornos cognitivos da comunicação. A Figura 1 apresenta os tipos de transtornos de linguagem oral incluídos neste modelo, os quais se encontram descritos detalhadamente a seguir, conforme a sintomatologia, etiologia e os correlatos neuroanatômicos.

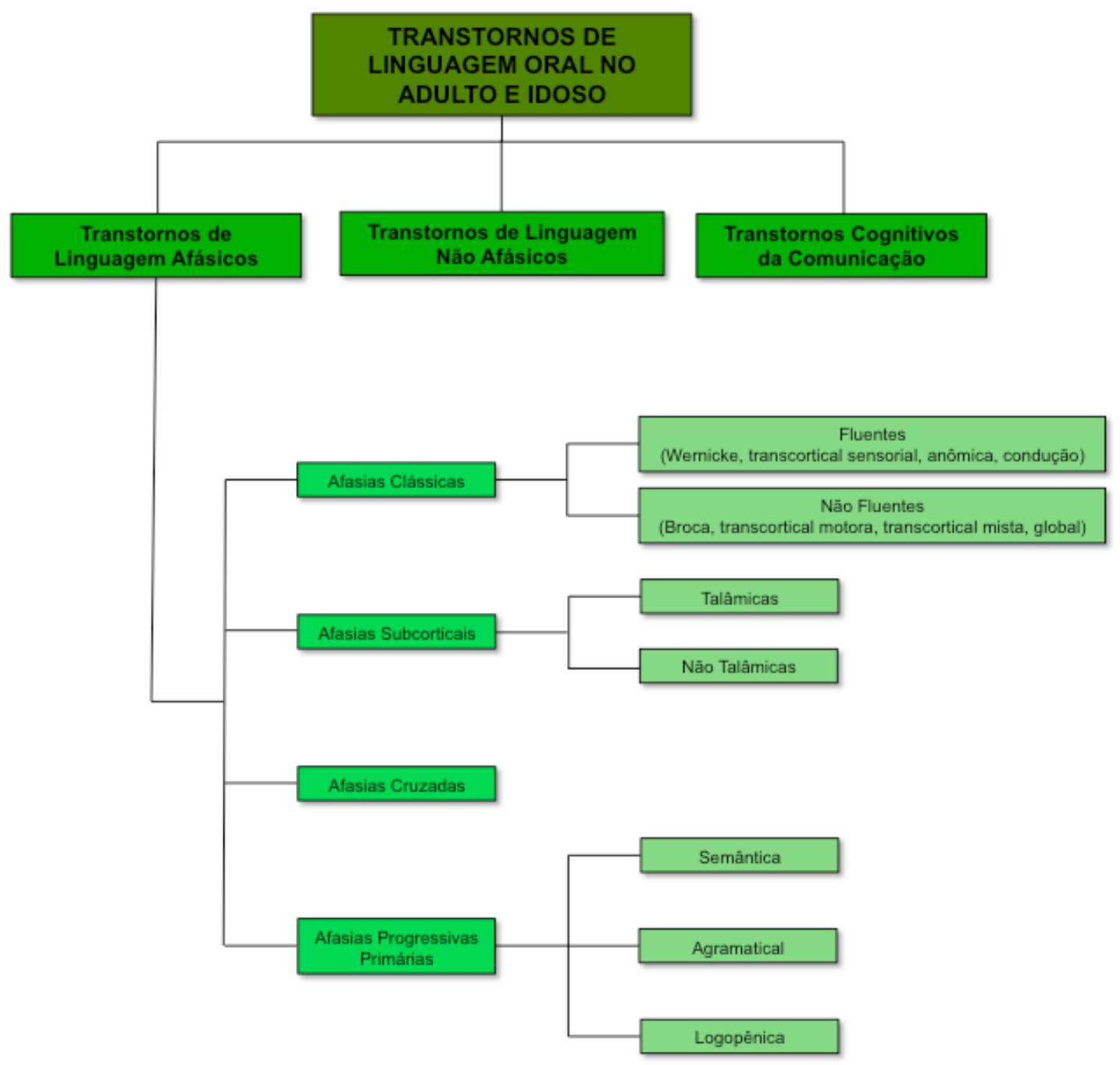

Figura 1. Classificação dos transtornos de linguagem oral em adultos e idosos

O adequado diagnóstico fonoaudiológico dos transtornos em questão se dá, primordialmente, através de uma avaliação compreensiva da linguagem e dos demais domínios da cognição, da história e diagnóstico médico do paciente, e de informações do exame de neuroimagem. A avaliação da linguagem deve incluir a investigação da fluência de fala, repetição, compreensão oral, nomeação oral, aspectos gramaticais, leitura e escrita, pragmática, prosódia, e das manifestações linguísticas. Os demais domínios cognitivos que devem ser investigados são a memória episódica, a memória de trabalho, as funções executivas, a atenção, o comportamento e as habilidades visuoespaciais. Os correlatos neurais são realizados com base nos dados de neuroimagem, que podem ser obtidos 
através de técnicas de tomografia computadorizada, ressonância magnética, tomografia por emissão de fóton único (SPECT), ou tomografia por emissão de pósitrons (PET), a depender da indicação médica para cada caso em específico.

A respeito do uso das terminologias das áreas cerebrais "área de Broca" e "área de Wernicke", devido a recomendações recentes da literatura em neurobiologia da linguagem, elas serão referidas por suas respectivas áreas neuroanatômicas, parte posterior do giro frontal inferior e giro temporal superior do hemisfério esquerdo, respectivamente. ${ }^{4}$

\section{Transtornos de linguagem afásicos}

Os transtornos de linguagem afásicos são alterações nos processos linguísticos expressivos e/ou compreensivos produzidos por lesões no SNC, que limitam significativamente a comunicação humana e se apresentam com outras funções cognitivas relativamente intactas. ${ }^{5}$ Podem ser subclassificados em afasias clássicas, afasias cruzadas, afasias subcorticais e APPs.

\section{Afasias clássicas}

A principal causa das afasias clássicas são as lesões cerebrais adquiridas, como o Acidente Vascular Encefálico (AVE) (que á causa mais frequente), o Traumatismo Cranioencefálico (TCE), os tumores e as infecções encefálicas. ${ }^{7}$ Diferentes subclassificações das afasias clássicas podem ser encontradas na literatura, sendo que uma das mais utilizadas é a que classifica estes transtornos em dois grandes grupos, as afasias fluentes (de Wernic- ke, transcortical sensorial, de condução e anômica) e as não fluentes (de Broca, transcortical motora, transcortical mista e global). ${ }^{2,6}$ No entanto, os mesmos tipos de afasias podem ser subclassificados em afasias perisilvianas (Broca, global, Wernicke, condução) e extraperisilvianas (transcortical motora, transcortical sensorial, mista transcortical e anômica) ${ }^{7}$ ou em emissivas (Broca, condução, transcortical motora), receptivas (Wernicke, transcortical sensorial, anômica) e mistas (transcortical mista, mista, global) ${ }^{8}$ A subclassificação que será utilizada aqui é originada dos estudos da Escola de Afasia de Boston, e divide os subtipos em afasias fluentes e não-fluentes. ${ }^{2}$

A Figura 2 representa um fluxograma didático que auxilia o fonoaudiólogo a definir o subtipo de afasia clássica, com base nas características da tríade fluência, repetição e compreensão da linguagem oral. Os demais aspectos de linguagem são importantes para o diagnóstico diferencial entre os subtipos. Deste modo, o Quadro 1 descreve detalhadamente cada subtipo das afasias clássicas. 2,6,9 É importante ressaltar que na prática clínica muito pacientes não se encaixam adequadamente em nenhum desses subtipos. Quando essa dificuldade de classificação ocorre, sugere-se que a avaliação fonoaudiológica objetive avaliar a natureza cognitiva do prejuízo de linguagem a fim de definir os domínios afetados que serão, então, os objetivos da reabilitação fonoaudiológica, mantendo como diagnóstico "transtorno de linguagem afásico não-especificado".

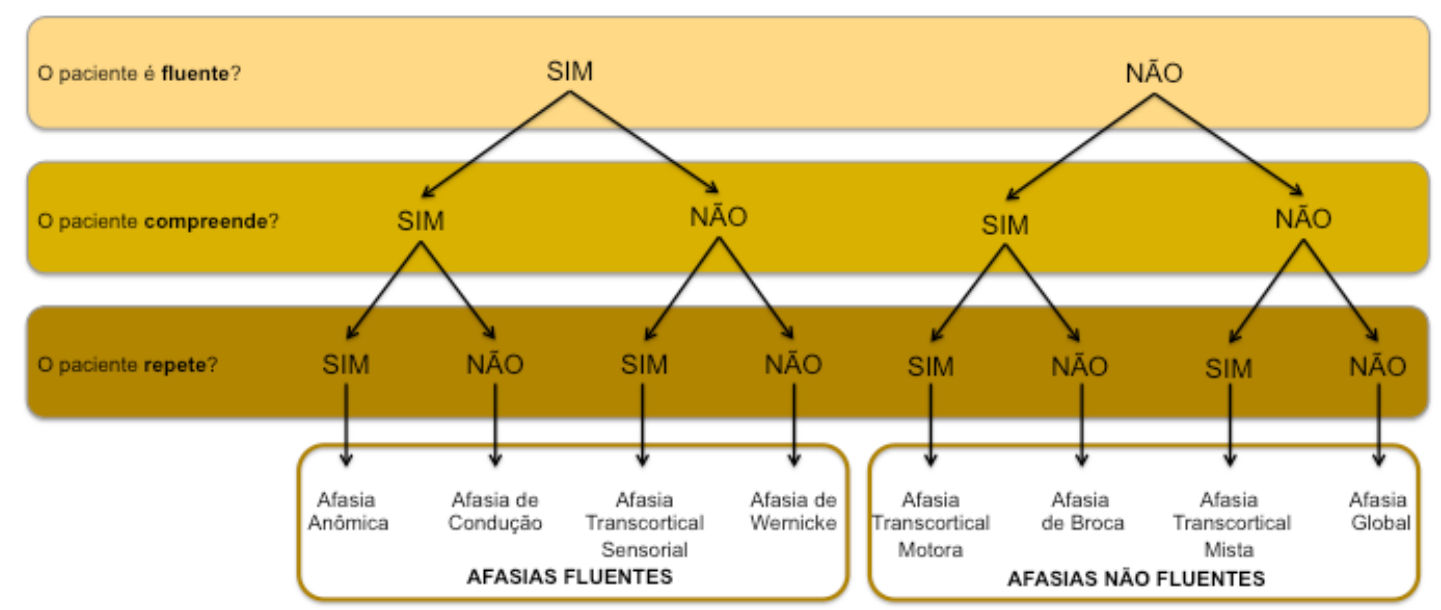

Figura 2. Fluxograma para classificação das afasias clássicas 
Quadro 1. Descrição das afasias clássicas

\begin{tabular}{|c|c|c|c|}
\hline & $\begin{array}{c}\text { Tríade fluência, repetição e } \\
\text { compreensão }\end{array}$ & \begin{tabular}{|c|} 
Outras características e \\
manifestações de linguagem
\end{tabular} & $\begin{array}{c}\text { Substratos Neurais no } \\
\text { Hemisfério Esquerdo }\end{array}$ \\
\hline \multicolumn{4}{|c|}{ Afasias Fluentes } \\
\hline $\begin{array}{l}\text { Afasia de } \\
\text { Wernicke }\end{array}$ & $\begin{array}{l}\text { Fluência: preservada } \\
\text { Repetição: prejudicada } \\
\text { Compreensão: prejudicada }\end{array}$ & $\begin{array}{l}\text { Jargão, logorréia, anomia, neo- } \\
\text { logismos, } \\
\text { circunlóquios, parafasias fonêmi- } \\
\text { cas e formais. }\end{array}$ & $\begin{array}{l}\text { Giro temporal superior, giro tem- } \\
\text { poral médio, lobo parietal inferior, } \\
\text { giro angular, giro de Heschl, polo } \\
\text { temporal, putamen. }\end{array}$ \\
\hline $\begin{array}{l}\text { Afasia de } \\
\text { Condução }\end{array}$ & $\begin{array}{l}\text { Fluência: preservada } \\
\text { Repetição: prejudicada } \\
\text { Compreensão: preservada }\end{array}$ & Parafasias fonêmicas e formais. & $\begin{array}{l}\text { Fascículo arqueado (especialmen- } \\
\text { te segmento posterior), áreas } \\
\text { corticais da região perisilviana } \\
\text { posterior (giro supramarginal } \\
\text { esquerdo e adjacências), giro } \\
\text { de Heschl. }\end{array}$ \\
\hline $\begin{array}{c}\text { Afasia } \\
\text { Transcortical } \\
\text { Sensorial }\end{array}$ & $\begin{array}{l}\text { Fluência: preservada } \\
\text { Repetição: preservada } \\
\text { Compreensão: prejudicada }\end{array}$ & $\begin{array}{l}\text { Parafasias verbais, fonêmicas e } \\
\text { semânticas, anomias, ecolalia, } \\
\text { neologismos. }\end{array}$ & $\begin{array}{l}\text { Proximidades da junção dos lobos } \\
\text { temporal, parietal e occipital, giro } \\
\text { temporal posterior médio, giro } \\
\text { temporal inferior, giro angular } \\
\text { inferior. }\end{array}$ \\
\hline $\begin{array}{l}\text { Afasia } \\
\text { anômica }\end{array}$ & $\begin{array}{l}\text { Fluência: preservada } \\
\text { Repetição: preservada } \\
\text { Compreensão: preservada }\end{array}$ & $\begin{array}{l}\text { Anomias, pausas frequentes, } \\
\text { substituições semânticas. } \\
\text { Maior dificuldade em determinada } \\
\text { categoria semântica. }\end{array}$ & $\begin{array}{l}\text { Não é confiavelmente associada } \\
\text { a uma região específica. Ano- } \\
\text { mias para substantivos podem } \\
\text { estar associadas a lesões no pólo } \\
\text { temporal, giro temporal médio e } \\
\text { inferior. Anomias para verbos as- } \\
\text { sociam-se a lesões no lobo frontal } \\
\text { (giro frontal inferior e conexões). }\end{array}$ \\
\hline \multicolumn{4}{|c|}{ Afasias Não Fluentes } \\
\hline $\begin{array}{l}\text { Afasia de } \\
\text { Broca }\end{array}$ & $\begin{array}{l}\text { Fluência: prejudicada } \\
\text { Repetição: prejudicada } \\
\text { Compreensão: preservada }\end{array}$ & $\begin{array}{l}\text { Fala lentificada e com esforço, } \\
\text { parafasias fonéticas e fonêmicas, } \\
\text { anomias, estereotipias verbais, } \\
\text { agramatismo, fala telegráfica, } \\
\text { alterações de prosódia. } \\
\text { Dificuldade de compreensão em } \\
\text { frases de maior complexidade } \\
\text { gramatical, e maior dificuldade } \\
\text { em emitir verbos do que subs- } \\
\text { tantivos. }\end{array}$ & $\begin{array}{l}\text { Parte posterior do giro frontal } \\
\text { inferior, opérculo frontoparietal, } \\
\text { e parte anterior da ínsula. }\end{array}$ \\
\hline $\begin{array}{c}\text { Afasia } \\
\text { Transcortical } \\
\text { Motora }\end{array}$ & $\begin{array}{l}\text { Fluência: prejudicada } \\
\text { Repetição: preservada } \\
\text { Compreensão: preservada }\end{array}$ & $\begin{array}{l}\text { Redução da fala espontânea e da } \\
\text { iniciativa de fala. } \\
\text { Nomeação melhor que a fala } \\
\text { espontânea. }\end{array}$ & $\begin{array}{l}\text { Lesões no córtex pré-frontal e } \\
\text { vizinhanças, com giro frontal } \\
\text { inferior preservado. }\end{array}$ \\
\hline \begin{tabular}{|c|} 
Afasia \\
Transcortical \\
Mista \\
\end{tabular} & $\begin{array}{l}\text { Fluência: prejudicada } \\
\text { Repetição: preservada } \\
\text { Compreensão: prejudicada } \\
\end{array}$ & $\begin{array}{l}\text { Expressão verbal severamente } \\
\text { prejudicada, anomias, ecolalias. }\end{array}$ & $\begin{array}{l}\text { Lesões corticais extensas que } \\
\text { mantêm o córtex perisilviano } \\
\text { preservado. }\end{array}$ \\
\hline $\begin{array}{l}\text { Afasia } \\
\text { Global }\end{array}$ & $\begin{array}{l}\text { Fluência: prejudicada } \\
\text { Repetição: prejudicada } \\
\text { Compreensão: prejudicada }\end{array}$ & $\begin{array}{l}\text { Prejuízo grave de todas modali- } \\
\text { dades da linguagem, emissão de } \\
\text { fala lenta e laboriosa, hesitações, } \\
\text { mutismo, anomia severa. } \\
\text { Frequentemente, a melhora do } \\
\text { quadro evolui para afasia de } \\
\text { Broca. }\end{array}$ & $\begin{array}{l}\text { Lesões corticais extensas que } \\
\text { comprometem grande parte das } \\
\text { regiões perisilvianas e suas cone- } \\
\text { xões subcorticais. }\end{array}$ \\
\hline
\end{tabular}

\section{Afasias subcorticais}

As afasias subcorticais são definidas como alterações da expressão e/ou compreensão da linguagem decorrentes de lesões em áreas subcorticais do cérebro, com as áreas corticais preservadas. A apresentação clínica é bastante heterogênea em termos de fenótipo linguístico e severidade. Além disso, as correlações anátomo-clínicas são menos consistentes do que nas afasias decorrentes de le- sões corticais e isso ocorre, principalmente, devido à imprecisão a respeito da função das estruturas subcorticais no processamento linguagem. Apesar dessas inconsistências, as afasias subcorticais parecem ter como característica em comum a repetição preservada. ${ }^{10}$

A classificação mais utilizada para as afasias subcorticais, divide-as em talâmicas e não-talâmicas. ${ }^{6}$ Nas primeiras, como o próprio nome refere, 
as lesões ocorrem no tálamo. O perfil linguístico depende da combinação do local de lesão e da topografia da linguagem dentro das inúmeras vias de conexões envolvidas. O mecanismo de diásquise cerebral pode ter um importante papel na apresentação clínica de lesões subcorticais talâmicas. ${ }^{6}$ A maioria dos estudos sobre as afasias talâmicas reportam prejuízos seletivos nas funções léxico-semânticas, com fluência de fala preservada. No entanto, achados menos frequentes também apontam ocorrência de parafasias fonéticas e algum prejuízo gramatical. ${ }^{6}$ Já nas afasias subcorticais não-talâmicas, as áreas afetadas são os núcleos da base. O perfil linguístico tende a ser caracterizado por prejuízo na fluência de fala, ocorrência de parafasias literais, e compreensão e nomeação preservadas. ${ }^{10}$ Mecanismos de hipoperfusão, dano isquêmico, desconexão e efeito de pressão podem estar associados com os achados clínicos deste subtipo de afasia subcortical, ${ }^{6,11}$ o que destaca a importância de uma interpretação cautelosa quanto as correlações anátomo-clínicas.

\section{Afasia Cruzada}

Mais de 95\% dos indivíduos destros têm o hemisfério esquerdo dominante para a linguagem. Nessa população, quando lesões cerebrais ocorrem no $\mathrm{HE}$ e atingem áreas cerebrais envolvidas no processamento da linguagem há a manifestação da afasia. No restante da população destra, menos de $5 \%$, o processamento da linguagem ocorre predominantemente no hemisfério direito (HD). Neste grupo de pessoas, lesões no HD levam a quadros de afasia, que são denominadas de afasias cruzadas. ${ }^{12}$ Assim, a definição de afasia cruzada pode ser resumida como sendo um transtorno afásico de linguagem, secundário a lesões no HD em indivíduos destros; ${ }^{12}$ ou um transtorno afásico decorrente de lesão cerebral unilateral do hemisfério ipsilateral à dominância manual do indivíduo. ${ }^{13}$

As afasias cruzadas correspondem a menos de $4 \%$ dos transtornos afásicos e aproximadamente $70 \%$ dos casos se manifestam como uma versão “espelhada' das afasias clássicas. ${ }^{5}$

\section{Afasias Progressivas Primárias}

A APP é um grupo de doenças neurológicas identificado por prejuízo seletivo de linguagem decorrente de um processo neurodegenerativo em regiões cerebrais frontais e temporais envolvidas no processamento da linguagem..${ }^{14} \mathrm{O}$ conhecimento que se tem desta doença progressiva e fatal é relativamente recente, e até o momento não há nenhum tipo de tratamento farmacológico para curar ou mudar o curso da doença. ${ }^{15}$ Apesar disso, há evidências de que tratamentos não-farmacológicos, como a terapia fonoaudiológica por exemplo, podem atrasar a evolução do transtorno de linguagem e melhorar a qualidade de vida dos pacientes. ${ }^{16,17}$

A primeira descrição de um caso de afasia decorrente de neurodegeneração foi feita por Arnold Pick em 1892. Aproximadamente um século depois, Marsel Mesulam descreveu uma série de casos de transtornos progressivos da linguagem, aos quais nomeou APP. Durante duas décadas, as APPs foram classificadas nos subtipos fluente (ou demência semântica) e não-fluente. ${ }^{18}$ Com o passar dos anos, percebeu-se que muitos casos não preenchiam os critérios para nenhum dos dois subtipos, e em 2004 Gorno-Tempini e seus colaboradores descreveram um terceiro subtipo de APP chamado afasia logopênica. Em 2011, foram publicados os critérios diagnósticos para as três variantes da APP, que são utilizados até o presente momento e estão descritas a seguir, conforme tal publicação. ${ }^{18}$

Variante semântica: a fala espontânea é fluente, mas há evidente anomia e dificuldade para compreender palavras isoladas. Nas fases iniciais, há a ocorrência de erros na nomeação que são mais frequentes em palavras pouco familiares e de baixa frequência, podendo ocorrer parafasias verbais e semânticas, circunlóquios, generalizações (ex.: falar "bicho" para qualquer espécie de animal) e omissões. Esta variante também se apresenta com dislexia e disgrafia de superfície, que são respectivamente a dificuldade de ler e escrever palavras irregulares devido ao comprometimento da rota lexical de leitura e escrita. Outros domínios cognitivos estão preservados, como a memória episódica, as praxias, as funções visuo-espaciais e as funções executivas. O quadro clínico é decorrente de atrofia no polo temporal do HE e/ou HD. Estudos post-mortem de pacientes com essa variante encontraram achados patológicos que indicam acúmulo de determinadas proteínas alteradas no cérebro, sendo as mais frequentes a degeneração frontotemporal pela proteína tau e pela proteína TDP, assim como achados patológicos de doença de Alzheimer (acúmulo da proteína beta-amilóide e emaranhados neurofibrilares de proteína tau).

Variante agramatical ou não-fluente: caracteriza-se principalmente por uma fala não-fluente. 
Há a ocorrência de apraxia de fala e agramatismo, que podem aparecer de forma isolada ou em conjunto. $\mathrm{O}$ agramatismo se manifesta pela emissão de sentenças simples e curtas, com alterações morfológicas em verbos e substantivos, e na ordem das palavras. Já a apraxia de fala se caracteriza pela fala lentificada; prosódia anormal; substituições, adições, repetições e/ou prolongamentos de sons da fala; e possível dificuldade para iniciar a emissão com tentativas e erros nos movimentos articulatórios. Esta variante tem como substrato neural atrofia nas regiões cerebrais frontais ou insulares do HE. O achado patológico mais frequente é a degeneração frontotemporal por alteração na proteína tau.

Variante logopennica: a variante logopênica foi descrita mais recentemente e caracteriza-se por dificuldade em repetir sentenças e para encontrar palavras (chamado efeito "ponta da língua"). Podem ocorrer erros fonológicos na fala, mas não há agramatismo ou dificuldade de compreensão oral. $\mathrm{O}$ achado clássico na neuroimagem de indivíduos com esta variante da APP é a atrofia na junção temporopariental do HE. Os achados patológicos são quase sempre de patologia da doença de Alzheimer, isto é, emaranhados neurofibrilares de proteína tau e placas senis pelo acúmulo da proteína beta amilóide.

\section{Distúrbio de linguagem não afásico}

O distúrbio de linguagem não afásico se caracteriza por uma performance normal ou próxima do normal em baterias de avaliação de afasia, apesar da ocorrência de déficits de linguagem em tarefas de linguagem de ordem superior ou alta complexidade. ${ }^{19}$ Complementarmente, é possível adicionar a esta definição dificuldades de linguagem sutis, muitas vezes perceptíveis pelo indivíduo e pelo avaliador, mas de difícil detecção na avaliação.

A etiologia pode ser diversa, podendo decorrer de distúrbios cerebrais progressivos ou não-progressivos, de lesões no HE ou no HD. ${ }^{19,20}$ Tais distúrbios são muito frequentes em casos de TCE, sendo que muitos casos apresentam quadros de afasia na fase aguda, mas progridem com melhora do quadro, permanecendo apenas com transtorno de linguagem não-afásico, mas que ainda limita a comunicação. ${ }^{21}$ Os déficits de linguagem mais frequentes em casos de TCE ocorrem em habilidades de linguagem de ordem superior, como as habilidades discursivas..$^{20}$ Os distúrbios não afásicos podem ocorrer em casos de doença de Alzheimer, manifestando-se principalmente através de déficits léxico-semânticos e de compreensão oral. ${ }^{22,23}$

É possível que nos casos de lesões no HE, as lesões estejam localizadas fora das principais áreas do processamento da linguagem, interrompendo as conexões com as redes de linguagem, ou que atinjam essas áreas em pequenas proporções. ${ }^{19}$ Nesses casos, as funções básicas da linguagem (processamento léxico-semântico, gramática, fonologia) podem estar alteradas, mas em um nível de severidade que não caracterize um quadro afásico.

Nos casos de transtornos de linguagem decorrentes de lesões no HD, em indivíduos que não possuem dominância para a linguagem neste hemisfério, os prejuízos acometem as funções de pragmática, prosódia, processamento léxico-semânticos, textual e discursivo. ${ }^{6,24}$ Pacientes com lesões de HD podem apresentar dificuldades para produzir e perceber a prosódia emocional e linguística, para realizar tarefas de julgamento semântico, e para compreender informações implícitas e conteúdos inferenciais. ${ }^{6}$

\section{Transtorno cognitivo da comunicação}

O transtorno cognitivo da comunicação é estabelecido quando as dificuldades de comunicação (expressão, compreensão, leitura, escrita, interação social) decorrem de déficits em funções cognitivas não-linguísticas, mas que intermediam o funcionamento da linguagem, como a memória, a atenção, e as funções executivas. Eles podem ocorrer em decorrência de qualquer condição neurológica e até psiquiátrica, e são comuns em quadros de demência, prejuízo cognitivo leve, AVE, tumores cerebrais e TCE. ${ }^{25-27}$

Para clarificar o conceito de transtorno cognitivo da comunicação, é possível tomar como exemplos os déficits nos seguintes domínios cognitivos e suas possíveis consequências na comunicação: memória episódica - o indivíduo não lembra que já fez determinada pergunta ou afirmação e repete-a constantemente, interferindo na efetividade da sua comunicação; atenção - quando o indivíduo não consegue manter a atenção na conversação, ocorre a perda de informação, podendo prejudicar a compreensão da mensagem. Neste caso, o déficit não é na função de compreensão oral, mas sim na atenção, o que pode ser facilmente confundido; controle inibitório - pode ter como consequência na comunicação dificuldades em respeitar os turnos de 
fala e emissões impróprias para o contexto social/ comunicacional em questão.

Apesar da doença de Alzheimer poder apresentar transtorno de linguagem não afásico, conforme descrito na seção anterior, também pode apresentar o transtorno cognitivo da comunicação ${ }^{25} \mathrm{~A}$ doença de Alzheimer caracteriza-se principalmente por prejuízo na memória episódica. Como a memória episódica é importante para a organização do discurso, há um impacto direto na comunicação. As principais características do transtorno de comunicação na doença de Alzheimer são: dificuldades de compreensão por não conseguir acessar as informações da conversa com as informações da memória episódica, repetição de sentenças e perguntas e dificuldade em lembrar o tópico da conversa. Tais dificuldades podem ocasionar problemas comportamentais como depressão, irritabilidade e até agressividade. O distúrbio cognitivo da comunicação também pode ocorrer em casos de demência vascular. ${ }^{25} \mathrm{~A}$ apresentação clínica da demência vascular depende das áreas afetadas, que podem ser diversas. Apesar disso, são comuns as dificuldades de atenção e de funções executivas. O impacto na comunicação se manifesta por redução na fluência de fala, dificuldade de compreensão por não conseguir armazenar a informação ouvida na memória de trabalho, e discurso confuso.

Nos casos de TCE, podem ocorrer dificuldades de memória episódica, atenção, memória de trabalho, velocidade de processamento da informação, funções executivas, cognição social e autoconsciência. ${ }^{28,29}$ Dificuldades discursivas podem então emergir, apresentando discurso com informação insuficiente, redundância de informação, falhas para estruturar o discurso, e colocação inadequadas para a situação. ${ }^{30}$

\section{Considerações Finais}

O presente manuscrito descreveu uma proposta de apresentação dos transtornos de linguagem oral que mais frequentemente acometem adultos e idosos, classificando-os em três grandes categorias. A primeira categoria é composta pelos transtornos de linguagem afásicos. Estes são caracterizados por alterações nos processos linguísticos, que limitam significativamente a comunicação e têm outras funções cognitivas intactas, em consequência a lesões no SNC. Dentro deste grupo estão as afasias clássicas, as afasias subcorticais, as afasias cruza- das e as APPs. A segunda categoria é o transtorno de linguagem não afásico, que é estabelecido quando há performance normal ou próxima do normal em baterias de avaliação de afasia, com déficits em tarefas de linguagem de ordem superior ou alta complexidade, ou com dificuldades de linguagem sutis. A terceira categoria, o transtorno cognitivo da comunicação, caracteriza-se por dificuldades de comunicação decorrentes de déficits em funções cognitivas não linguísticas, em decorrência de condições neurológicas ou psiquiátricas.

A utilização desta classificação tem como objetivo facilitar a comunicação entre os profissionais, permitir a caracterização dos pacientes e facilitar o raciocínio clínico. No entanto, é sabido que muitos casos não são possíveis de serem classificados, por apresentarem características comuns a mais de um tipo de transtorno, ou por serem casos atípicos. A impossibilidade de classificação de um quadro de transtorno de linguagem, não impede um adequado planejamento terapêutico e sua execução. Nestas situações, os resultados da avaliação fonoaudiológica devem nortear a reabilitação, independentemente do diagnóstico fonoaudiológico. As habilidades linguísticas e/ou cognitivas deficitárias poderão ser objetivo da reabilitação, enquanto que as habilidades preservadas poderão ser utilizadas na terapia como facilitadores.

É esperado que o avanço científico na área permita um melhor entendimento dos transtornos de linguagem, das doenças neurológicas e da própria neurobiologia da linguagem. Naturalmente, este avanço científico poderá levar a mudanças na classificação destas condições e no próprio paradigma de estabelecimento dos diagnósticos médico e fonoaudiológico.

\section{Referências bibliográficas}

1. Damásio AR. Signs of aphasia. In: Sarno MT. Aquired aphasia. 3rd ed. San Diego: Academic Press; 1998. 687 p.

2. Kemmerer D. Cognitive neuroscience of language. New York: Psychology Press; 2015. 623 p.

3. Brandão L, Fonseca RP, Ortiz KZ, Azambuja D, de Salles JF, Navas AL, et al. Neuropsychology as a specialty in Speech Language and Hearing Sciences: Consensus of Brazilian Speech Language Pathologists and Audiologists. Distúrb Comun. 2016; 28: $378-87$.

4. Tremblay P, Dick AS. Broca and Wernicke are dead, or moving past the classic model of language neurobiology. Brain Lang. 2016; 162: 60-71. 
5. Davis GA. Aphasiology: Disorders and Clinical Practice. 2nd ed. Boston: Pearson; 2007.

6. Stemmer B, Whitaker HA. Handbook of neuroscience of language. London: Elsevier; 2008. 490 p.

7. Benson DF, Ardilla A. Aphasia: A Clinical Perspective. Oxford University Press; 1996. 441 p.

8. Ortiz KZ. Distúrbios Neurológicos Adquiridos Linguagem e Cognição. Manole; 2010. 484 p.

9. Yourganov G, Smith KG, Fridriksson J, Rorden C. Predicting aphasia type from brain damage measured with structural MRI. Cortex. 2015; 73: 203-15.

10. Kuljic-Obradovic DC. Subcortical aphasia: three different language disorder syndromes? Eur J Neurol. 2003; 10: 445-8.

11. Hillis AE, Barker PB, Wityk RJ, Aldrich EM, Restrepo L, Breese EL, et al. Variability in subcortical aphasia is due to variable sites of cortical hypoperfusion. Brain Lang. 2004; 89: $524-30$

12. Kim WJ, Yang EJ, Paik N-J. Neural Substrate Responsible for Crossed Aphasia. J Korean Med Sci. 2013; 28: 1529.

13. Mastronardi L, Ferrante L, Maleci A, Puzzilli F, Lunardi P, Schettini G. Crossed aphasia. An update. Neurosurg Rev. 1994; 17: 299-304.

14. Mesulam MM. Primary progressive aphasia. Ann Neurol. 2001; 49: 425-32.

15. Tsai RM, Boxer AL. Treatment of frontotemporal dementia. Curr Treat Options Neurol. 2014; 16: 319.

16. Cadório I, Lousada M, Martins P, Figueiredo D. Generalization and maintenance of treatment gains in primary progressive aphasia (PPA): a systematic review: Treatment gains in PPA: a systematic review. Int J Lang Commun Disord. 2017; 52: 543-60.

17. Tippett DC, Hillis AE, Tsapkini K. Treatment of Primary Progressive Aphasia. Curr Treat Options Neurol [Internet]. 2015 [cited 2018 Jan 6];17. Available from: http://link.springer. com/10.1007/s11940-015-0362-5

18. Gorno-Tempini ML, Hillis AE, Weintraub S, Kertesz A, Mendez M, Cappa SF, et al. Classification of primary progressive aphasia and its variants. Neurology. 2011; 76: $1006-14$

19. McDonald S. Viewing the brain sideways? Frontal versus right hemisphere explanations of non-aphasic language disorders. Aphasiology. 1993; 7: 535-49.
20. Vas AK, Chapman SB, Cook LG. Language impairments in traumatic brain injury. In: Handbook of Clinical Neurology [Internet]. Elsevier; 2015 [cited 2018 Jan 6]. p. 497-510. Available from: http://linkinghub.elsevier.com/retrieve/pii/ B9780444635211000315

21. Prigatano GP, Roueche JR, Fordyce DJ. Nonaphasic language disturbances after closed head injury. Lang Sci. 1985; 7: 217-29.

22. Klimova B, Maresova P, Valis M, Hort J, Kuca K. Alzheimer's disease and language impairments: social intervention and medical treatment. Clin Interv Aging. 2015; 10: $1401-8$.

23. Verma M, Howard RJ. Semantic memory and language dysfunction in early Alzheimer's disease: a review. Int J Geriatr Psychiatry. 2012; 27: 1209-17.

24. Cummings L. Pragmatics and Adult Language Disorders: Past Achievements and Future Directions. Semin Speech Lang. 2007; 28: 096-110.

25. Bayles KA, Tomoeda CK. Cognitive-communication disorders of dementia. Definition, diagnosis, and treatment. San Diego: Plural Publisching Inc.; 2014. 271 p.

26. Hewetson R, Cornwell P, Shum D. Social participation following right hemisphere stroke: influence of a cognitivecommunication disorder. Aphasiology. 2018; 32: 164-82.

27. Togher L, Wiseman-Hakes C, Douglas J, Stergiou-Kita M, Ponsford J, Teasell R, et al. INCOG Recommendations for Management of Cognition Following Traumatic Brain Injury, Part IV: Cognitive Communication. J Head Trauma Rehabil. 2014; 29: 353-68.

28. Gardner RC, Langa KM, Yaffe K. Subjective and objective cognitive function among older adults with a history of traumatic brain injury: A population-based cohort study. Brayne C, editor. PLOS Med. 2017; 14: e1002246.

29. Azouvi P, Arnould A, Dromer E, Vallat-Azouvi C. Neuropsychology of traumatic brain injury: An expert overview. Rev Neurol (Paris). 2017; 173: 461-72.

30. Snow P, Douglas J, Ponsford J. Conversational assessment following traumatic brain injury: a comparison across two control groups. Brain Inj. 1997; 11: 409-29. 